\title{
Paper Empirical Approach in Topology Control of Sensor Networks for Urban Environment
}

\author{
Bartosz Musznicki \\ Chair of Communication and Computer Networks, Poznań University of Technology, Poznan, Poland
}

https://doi.org/10.26636/jtit.2019.129918

\begin{abstract}
Research into the topology control of Wireless Sensor Networks (WSNs) is geared towards modeling and analysis of methods that may be potentially harnessed to optimize the structure of connections. However, in practice, the ideas and concepts provided by researchers have actually been rarely used by network designers, while sensor systems that have already been deployed and are under continued development in urban environments frequently differ from the patterns and research models available. Moreover, easy access to diversified wireless technologies enabling new solutions to be empirically developed and popularized has also been conducive to strengthening this particular trend.
\end{abstract}

Keywords-empirical approach, node deployment, sensor network, topology control, urban environment, WSN.

\section{Introduction}

The need to use devices that provide measurement data and those that transport thus obtained information to a destination point usually located deep inside the network is an inextricable element defining the operation of any Wireless Sensor Network (WSN) [1]. Hence, with reference to WSN, a sensor is typically understood not only as a component that performs measurements, but also as an entire small-size network node. This small and highly specialized microcomputer should be equipped with measurement sensors, but also with its own power supply, a wireless communication module, a microcontroller or microprocessor and memory [2]. In addition, other components defining the applications area, e.g. a GPS signal receiver or relays that make the control of external actuators possible [3], may be required as well.

Initially, work on sensor networks involved the individual authors' own hardware designs, mainly due to the lack of commercial availability of dedicated products. As recently as 5-10 years ago, sensor platforms belonging to the MICA2, MICAz and TelosB families were considered to be the most advanced and were most widely accepted by researchers. Over the past few years, general-purpose embedded platforms, such as the Arduino UNO, equipped with the ZigBee module, or the Raspberry Pi 3 Model B which provides IEEE $802.11 \mathrm{~b} / \mathrm{g} / \mathrm{n}$ and Bluetooth 4.1 wireless connectivity, seem to be used increasingly frequently. Because of their easy availability, affordable pricing and numerous configuration options, they enable researchers, enthusiasts and innovators alike to design and deploy sensor networks or sensor-like networks [4]-[8].

Another factor that supports the development and implementation of WSNs is the increasing coverage ensured by different wireless access networks [9]-[12] that can be used to transfer data. Moreover, Low-Power Wide-Area Network (LPWAN) technologies that support long-range low bit rate and energy efficient communication in sub- $1 \mathrm{GHz}$ frequencies are being developed (e.g. by LoRa Alliance, SigFox and Weightless SIG). In urban environments, such network infrastructure may be found, most frequently, on top of high-rise structures (see Fig. 1), i.e. mounted on masts or placed on rooftops of buildings, as well as at more unusual locations such as, for instance, on the branches of an artificial tree on the slope of a hill or in palm trees. Furthermore, components providing access to different local (short-range) wireless networks are common inside office and apartment buildings as well.

What becomes more and more apparent is the fact that objects which at first glance are a far cry from simple sensor nodes, here understood as devices with limited computational capabilities and battery-based power supply, are now being equipped with integrated sensor capabilities. In real-life applications, sensor functions are performed, ever more frequently, by vehicles and consumer devices, such as residential water meters, mobile phones and sports watches. They are often called smart objects and are considered to be capable of providing additional functionalities. Objects such as these may be combined to form an integrated system and are capable of cooperating to complete more complex and context-related tasks [13], [14]. They are often combined with additional analytical tools and distributed resources provided by cloud computing [15]. The ever-increasing potential in terms of the range of applications for sensor-based or sensor-like devices, which are already perceived as one of the components of the Internet of Things (IoT), is followed, within the domains of research and product marketing alike, by the need for their further differentiation. Subsequent subcategories with their particular functionalities and purposes clearly identified emerge [16], [17], including Vehicular Sensor Net- 


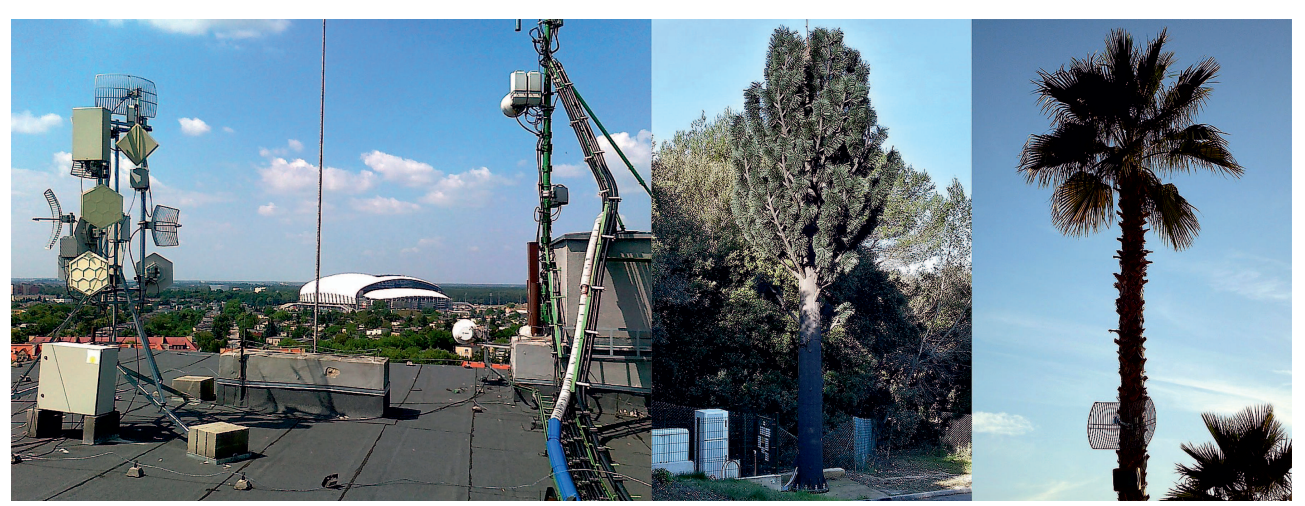

Fig. 1. A rooftop cellular and IEEE 802.11 networks base station (left) (Poznań, Poland, July 2011), an artificial tree used as a base station on a hillside (middle) (Sophia Antipolis, France, March 2016), a wireless station on a palm tree (right) (Athens, Greece, October 2013).

works (VSNs), Body Area Networks (BANs), home automation, smart factory or smart city, just to name a few. All of them, however, have one thing in common - they perform measurements and rely on wireless communication solutions [18].

In recent developments, attention is attached not only to functionality-related issues, but also to considerations related to the nature of the processes involved and to system of connections. The structure of connections and the way they are used depend on the environment and the conditions in which the network is operating, as well as on the assumptions made and the tasks that are to be performed. Such circumstances are often investigated in simulations of the required type [19]. It may be stated, based on the author's research, that the implementation phase is of a dual nature. It is sometimes preceded by a long-term research study (lasting for years), but more often simply follows the product development stage. This leads to a certain dissonance between research and actual implementation practices. On the one hand, the results of novel research related to optimization methods can be relied upon [20]. On the other, however, in response to changeable business needs, well-established technologies and solutions are continuously used, while the very vision of the sensor network is either being simplified or modified so that it meets the requirement of quick execution of ideas and commercial adaptation of the product to the needs of the market.

The above observation partly coincides with the opinion, as presented in [21], that "although new topology control algorithms are presented on a regular basis, topology control has never made the breakthrough in real-world deployments", and may be accompanied by a statement that the obstacles faced include the following: unrealistic assumptions, unsuitable graph structures, application agnosticism, unclear role in the stack and insufficient framework support. The arguments presented included insufficient utilization of graph-based methods for optimization of the structures of typical WSNs. In the author's opinion, with real-life implementations of sensor-type networks, it is rather the market situation and the way in which innovative products and services are created that is largely decisive for the approach adopted with regard to topology issues. As a consequence of this attitude, this paper provides a juxtaposition between theoretical and research-based views on the manner in which the structures of WSNs are managed on the one hand, and the empirical approach to deployment and implementation of sensor-based systems, as seen by a network architect with practical experience, on the other. The presentation is based on the author's experience with various networks and his involvement in tests pertaining to the networks under scrutiny.

Section 2 provides a definition of the notion of topology control, whereas Section 3 presents two types of physical arrangements of nodes that may be found in practice. Both categories are illustrated with examples of real systems. Conclusions are presented in Section 4.

\section{General Objectives of Network Topology Control}

Network topology is typically understood to be a model that describes the structure of connections between the elements within a given network [22] and is frequently presented in the form of a graph $\mathscr{G}=(\mathscr{V}, \mathscr{E})$, where $\mathscr{V}$ denotes the set of vertices (in other words nodes, such as sensors), whereas $\mathscr{E}$ is the set of edges (i.e. connections) between vertices [23]. This notation may refer to both physical relations (relative arrangement of nodes and connections between them, directly stemming from the properties of the transmission medium applied) and logical relations (an operational configuration based method for transmitting data via the network from the starting point to the end point, between the elements of the network's infrastructure [24]. As a result, physical and logical topologies may be distinguished [25]. Topology control is a related notion and in general encompasses different aspects related to planning, maintenance and adaptation of the system of connections within a given network [26]. Topology management is an alternative term used on some occasions [27], [28]. 
In its broadest and the most diverse scope, topology control is closely connected with wireless networks, not only due to the variable character of radio communications, but also due to the particular features of devices that rely on wireless transmission. Topology control is distinctively illustrated with regard to the ad-hoc networks [29]-[31], and becomes of special significance within the WSN context. As the development of sensor networks progresses, this issue is gaining in importance and reflects the increasingly more detail-oriented and extensive scrutiny of each of the aspects influencing the network structure.

Santi states that "topology control is the art of coordinating nodes' decisions regarding their transmitting ranges, in order to generate a network with the desired properties, e.g. connectivity, while reducing node energy consumption and/or increasing network capacity" [26]. Labrador and Wightman point out, more broadly, that "topology control is the reorganization and management of node parameters and modes of operation from time to time to modify the topology of the network with the goal of extending its lifetime while preserving important characteristics, such as network and sensing connectivity and coverage" [32]. At the same time, they emphasize that the above definition refers not only to the control of the transmitting power of sensors, but also to turning on and shutting off nodes depending on current needs. Aziz et al. provide the following definition "topology control is a technique that uses any controlled network parameter to generate and maintain a topology for the benefits of reducing energy consumption and achieving a desired property for an entire network" [33]. Li et al. present, in turn, a view that topology control is a fundamental benchmark "which characterizes how well a sensing field is monitored and how well each pair of sensors is mutually connected in WSNs" [34]. Consequently, studies related to topology control may include investigations into operational management and transmitting power control of radio modules [35], [36], energy-harvesting [37], interference prediction [38], as well as sensor placement [39], network coverage [40], logical network structure and message routing [41], [42], node functional diversification and hierarchy (e.g. chaining [43], and clustering [44]).

Having closely examined the development of this particular domain and taking advantage of the definitions presented above, it can be generalized that the term topology control covers all activities intended to influence the physical or logical structure of a network in order to optimize the way the network executes its tasks while retaining the expected properties.

\section{Sensor Nodes Deployment}

Node deployment is the basic element that influences the way the topology of a sensor network is controlled [1], [45]. Moreover, the empirical practices related to topology control in commercially-oriented ventures, as discussed in the following sections, seem to focus, first and foremost, on the distribution of nodes.
In real applications, depending on particular requirements or environmental conditions in which a given sensor network operates, random [40] or deterministic [46] distribution of network nodes may be distinguished. This distribution may be predicted at the designing stage, or can be partly or totally random in a dynamically changing working environment. In many real applications it is difficult or even impossible to assign a given type of node distribution within a network (or a part thereof) to just one of two categories. One should not forget that each sensor structure is characterized by a certain degree of determinism (therefore also a degree of randomness) that will vary along with changes in the external environment, though frequently to an extent that can be neglected in a given application.

The following subsections discuss both types of node deployment schemes that may be encountered in urban environments, i.e. random deployment and deterministic deployment, and provide examples of their implementations.

\subsection{Random Deployment}

Since the very beginning of work on WSNs, a general view has prevailed in the literature of the subject that random deployment of nodes [40] is the fundamental approach, serves as the point of departure while constructing WSNs and is typical of this group. One of the basic areas of application for such a network is monitoring the parameters of the natural environment (e.g. temperature or pressure) [47]. Much attention has been then given to methods for random deployment of sensors, while one of the most frequently mentioned examples illustrating the above would be a situation in which sensor devices are dropped from an aircraft over the area to be monitored [48].

The sheer multitude of potential applications of sensor networks that has been identified over nearly two past decades has led to numerous complex concepts related to the construction of the networks' physical topologies. Many of them depart from randomness of node deployment, understood in the direct and unconditional manner, by introducing some kind of order. In the author's opinion, one of the most interesting scenarios is a network in which the sensors are deployed in an unknown working environment, with the process carried out according to a predefined algorithm and based on information obtained during actual deployment [49].

Other networks that should also be noted within this context include VSNs, in which a sensor network typically covers the intended area - a road and its closest surroundings - and may be spread over tens or even hundreds of kilometers. In the case of such networks, one may speak of a combination of randomness and determinism, i.e. a certain portion of the sensors are deployed permanently alongside the road, while the sensors that communicate with them are those that may be deployed in vehicles. Their distribution is random in such a case and they frequently remain beyond the control of sensor networks' operators [50]. Vehicles may be then viewed as mobile agents that perform not only tasks assigned to them, but also 
additional functions that relate to stationary sensors [51]. Currently, it is rare that nodes in such systems communicate between one another, because typically the exchange of information is performed with a central point only (e.g. a control center or a data collection software). Furthermore, in the case of VSN, it is common to omit issues related to limitations of energy sources or computational power - so important in traditional WSNs [52]. This is why the largest number of implemented examples of sensor-type and sensor-like networks may be found in the group of systems related to transport and presented in the subsequent subsections. It is worthwhile noticing, at this point, that the largest systems of this type, based on smartphones that serve as mobile agents, form today's most widely used sensor networks and that their number and scope of functionalities are depicted by a continuous upward trend.

\subsubsection{Mobile Measurement Agent within INEA Network}

Following the research studies initiated by the author and carried out together with the associates from INEA, a regional Polish telecommunications operator, it was possible to perform measurements related to the operation of radio networks that are based on the IEEE 802.11 family of communication standards [53]. A detailed description of the tests and an analysis of the results obtained are presented in [11], while the conclusions from this work are presented below.

The first group of experiments was performed using access points providing wireless Internet access, with the use of the $2.4 \mathrm{GHz}$ band, to the passengers of 330 public transport vehicles in Poznań and Konin, two cities in the Greater Poland region. The movement of those agents was beyond any control of the telecommunications network operator because it was the transport operator that decided about the movement of the vehicles involved, at the same time impacting the topology of the network. By relying on devices known as RouterBoard RB751U, deployed in trams and buses and equipped with a $2.5 \mathrm{dBi}$ antenna and a $4 \mathrm{G}$ cellular network modem-based uplink (providing Internet connection), a collection of samples was performed in each vehicle once every 15 minute. The important parameters included noise floor level (background noise), expressed in $\mathrm{dBm}$, and the values of the Received Signal Strength Indicator (RSSI) related to each of the connected users were recorded. In this way, reliable around-the-clock distributions for the urban environment were obtained, thus allowing a technical and a business analysis useful for INEA to be performed.

The around-the-clock distribution of the average noise floor that occurred over the period of one month was particularly interesting. In order to verify the distributions observed, they were compared with the results obtained with the help of 10 stationary INEA access points operating in the $5 \mathrm{GHz}$ band, located on masts and on rooftops. It turned out that both trends were nearly identical, which is clearly visible

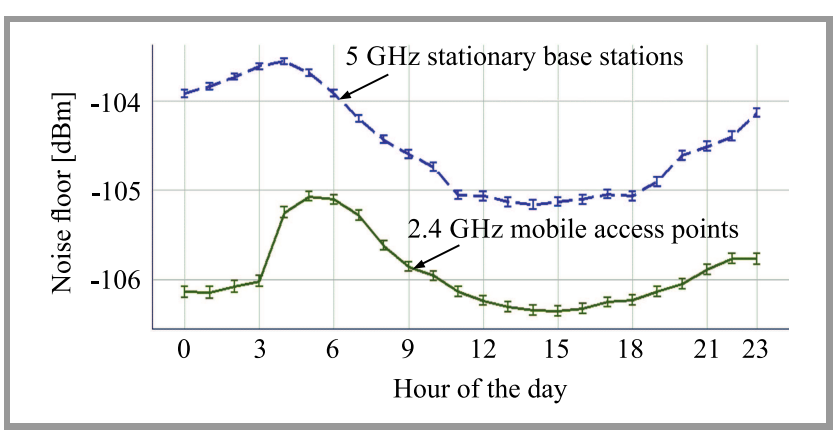

Fig. 2. Average noise floor observed with the use of INEA's IEEE 802.11 access points.

in Fig. 2. Each point in the graph corresponds to the average value from a given month, whereas confidence intervals correspond to standard deviation. Because the comparison involved a mobile network and a stationary network, both operating in different environmental conditions and with the application of antennas with a much higher gain and on other frequencies, the conclusion drawn is the changes in noise floor were mainly caused by external factors and were not typical of human activity. Further studies were conducted in 2017 with the use of a stationary $3.5 \mathrm{GHz}$ IEEE 802.16e WiMAX network, yielding similar characteristics and suggesting that they may result, in addition to other factors that are yet to be identified, from ambient temperature [54] changes.

The other group of experiments involved issues related to the operation of more than 20 thousand fixed residential WiFi hotspots, i.e. IEEE 802.11 access points that were located at INEA customers' homes. Each INEA subscriber takes advantage of a community Wi-Fi service based on home routers connected to the INEA_HotSpot_WiFi network.

The experience of mobile users was verified using a smartphone equipped with an IEEE $802.11 \mathrm{a} / \mathrm{b} / \mathrm{g} / \mathrm{n} / \mathrm{ac}(2 \mathrm{x} 2$ MIMO) radio module, GPS and GLONASS navigation receiver, as well as measurement and communication freeware. The test to be carried out involved measurements of signal parameters and extraction of technical information for each Basic Service Set Identifier (BSSID) observed. During the test, the user was moving, with the smartphone, on the sidewalk, between multi-story apartment buildings in council housing estates built in the 1970s. It was observed that 313 out of 1874 BSSIDs used the Service Set Identifier (SSID) with the name INEA HotSpot WiFi. The tests performed indicate that $59 \%$ of INEA residential hotspots could have been used for conversations with the use of Voice over IP (VoIP), provided that the user standing on a sidewalk was connected to the access point and that the strength of the signal received was not lower than $-75 \mathrm{dBm}$.

\subsubsection{Yanosik Driving Assistant and notiOne Location Beacon}

The development of mobile radio-location systems, as well as increasingly common packet data transmission has led 


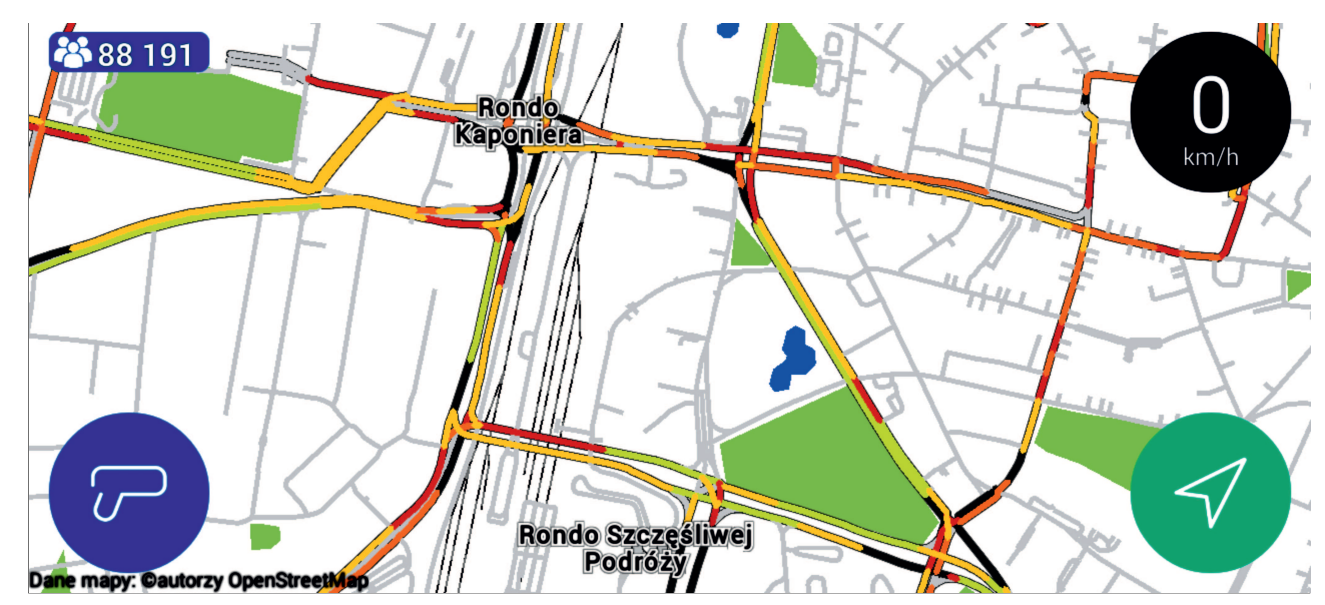

Fig. 3. Poznań city center road traffic map presented by Yanosik (ver. 3.1.1.1), 9 May 2017.

to widespread use, among drivers, of sensor systems based on mobile agents. No statistical comparisons are available that would illustrate the popularity of particular solutions, but, based on market observations, one may come to a conclusion that the Yanosik driving assistant, operated by a Poznań-based company named Neptis, is the leading platform among Polish drivers. On the global scale, such applications as Google Maps, HERE WeGo and Waze are based on similar concepts.

The Yanosik driving assistant is primarily a system used for exchanging information and warnings between drivers, relying on dedicated devices and smartphones (a special free app has to be installed). Both devices need to be wirelessly connected to the Internet (usually via a cellular network) to act as mobile agents. Vehicles (devices) do not communicate directly with other road users because the entirety of the exchange of information is performed on the central operator's host platform. This network has a dynamically variable random topology of the logical star type, in which the location of nodes depends on the situation on the road.

Drivers who report events, such as road accidents, road works or a police patrol, are the source of information. Along with a report, additional information is forwarded on the user's location. A smartphone with the Yanosik app may be also used as a navigation system with real-time traffic service. As indicated in a release issued to the author by Neptis in April 2017, at its peak times, the system is used by over 150,000 concurrent users, which means that it comprises the same number of sensor nodes. The further processing of data makes it possible to develop real time traffic intensity maps (Fig. 3). In addition, the author was shown the results of an investigation that demonstrated the use of information gathered from sensors embedded in smartphones (accelerometers and gyroscopes) in order to evaluate the quality of roads and driving comfort. This enabled data on vibrations experienced by drivers and vehicles, while in motion, to be analyzed. The smoothness of traffic flow and the average speed were studied as well.
In more complex network topologies, smartphones offer wireless interfaces of different types and make it possible to connect with devices of other types to execute additional agent functions. This provides a basis for the operation of devices known as notiOne, which are the so-called beacons (see Fig. 4). These simple and small-scale mobile transmitters broadcast signal that includes a device identifier and may be received by smartphones that happen to be in the vicinity. In this way, the location of the beacon is approximated based on the accurate location of the smartphone that received the beacon's signal. Effectiveness and accuracy of such geolocation depends on the number of nearby smartphones on which software co-operating with the system has been installed.

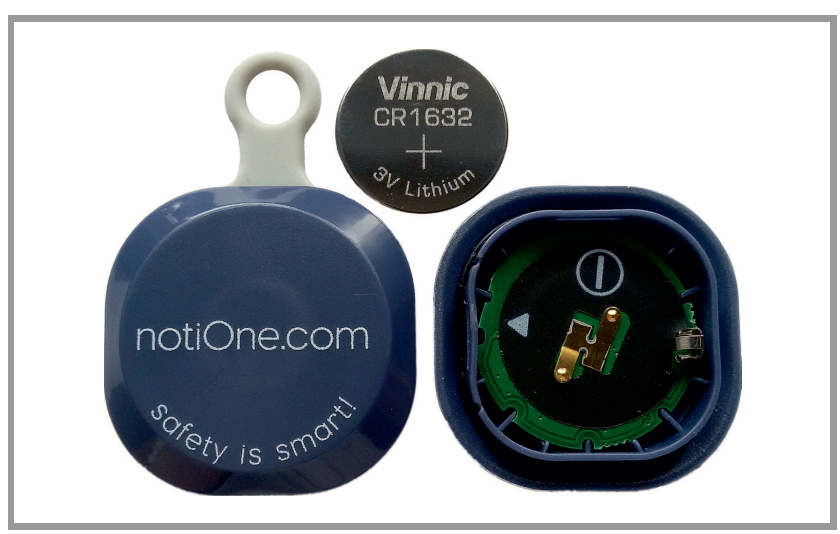

Fig. 4. Opened notiOne beacon with a CR1632 button cell battery.

The beacon may be attached to a key pendant or a dog collar, so that if lost, it makes it possible to easily obtain information on its location by means of a dedicated app. The location highlighted on the map will indicate the spot where the beacon's signal was received for the last time. The notiOne device is powered by a button cell battery that allows the device to work for nearly one year, with the range up to $90 \mathrm{~m}$ using Bluetooth 4.0 Low Energy connectivity. 


\subsubsection{The Veniam System}

The activity of Veniam serves as a good example of a successful implementation of research work on mobile sensor networks in a commercial product. The company's founders, Barros, Cardote, and Sargento, were previously involved in research on mobile and sensor networks, among others [55]-[58]. In 2003, as a result of their work, commissioned by city authorities, Veniam launched in Porto, Portugal, a mobile wireless network targeting to collect the results of measurements performed in the urban environment and improving the operational efficacy of the city's transport and service utility vehicles. Recently, the network comprised nearly 350 vehicles (buses, police cars, garbage trucks, taxi cabs) [59]. These vehicles are equipped with a device that serves as an access point (with a network interface, etc.) known as NetRider. To provide connectivity, radio base stations are used, operating in the bandwidth of $5.9 \mathrm{GHz}$ according to the IEEE $802.11 \mathrm{p}$ standard for the mobile environment, in particular for the so-called Intelligent Transport Systems (ITS) [60]. Each access point serves as an IEEE 802.11g Wi-Fi hotspot and makes the Internet available to passengers. Sensors are placed inside vehicles to make environmental measurements and to monitor the fleet. The vehicles may communicate with one another and serve as mobile transceivers (called mobile relays) [28], making indirect, real time communication between the control system and the out of range vehicles possible. In turn, when communication in real time is not possible or suffers from delays, thus forming, in fact, a delay tolerant network (DTN), a function of data extraction and temporary buffering, most frequently called data MULE (Mobile Ubiquitous LAN Extension) [61], is performed. If a vehicle that passed near a stationary sensor located at the edge of the road is just outside the base station coverage, the reading is taken (receiving a portion of data using IEEE 802.11 or Bluetooth) by using the local memory of the mobile agent, with data forwarded to the central repository once the connection with the base station has been reestablished [62]. The system employs a complex and variable topology of con- nections that is successfully created by means of different models and methods for wireless communication.

The author has had a chance to examine the Veniam network's control and management panel. The virtual environment software makes it possible to monitor the network in real time and to store and analyze the data extracted. The location of any vehicle is presented, just as are the estimated range of the hotspot and the live traffic intensity map for the area covered.

\subsubsection{Automated Road Passenger Transport}

Transport automation is another area of sensor applications that is currently under development. One of the leading European projects in this area was CityMobil2, launched in 2012 and concluded in August 2016, co-financed under the European Union's Seventh Framework Programme [63]. The goal was to create a pilot automated passenger road transport platform with automated and autonomous vehicles, and to carry out tests in a number of European urban environments [64]. The platform was made up of electric vehicles, i.e. mini buses equipped with wireless interfaces for communication with the control center, as well as with sensors necessary for the unmanned vehicles to operate. The entire system used a central controller that gathered data transmitted by the vehicles and controlled the vehicle movement, hence the physical network topology. In addition, the personnel was capable of override the control system.

In 2016, the author had a chance to visit a demonstration route used by autonomous vehicles in the French Sophia-Antipolis technology park, and took a ride in the EasyMile EZ10 autonomous vehicle shown in Fig. 5. The one-kilometer test lane with five stops was used by three autonomous shuttle service vehicles, each with the capacity of 9 passengers. By using a GPS receiver, proximity detectors and accelerometers, the vehicles were capable of adjusting the driving speed to other road users and avoided obstacles or, alternatively, stopped before them if avoiding the obstacle was impossible. When this was the case,

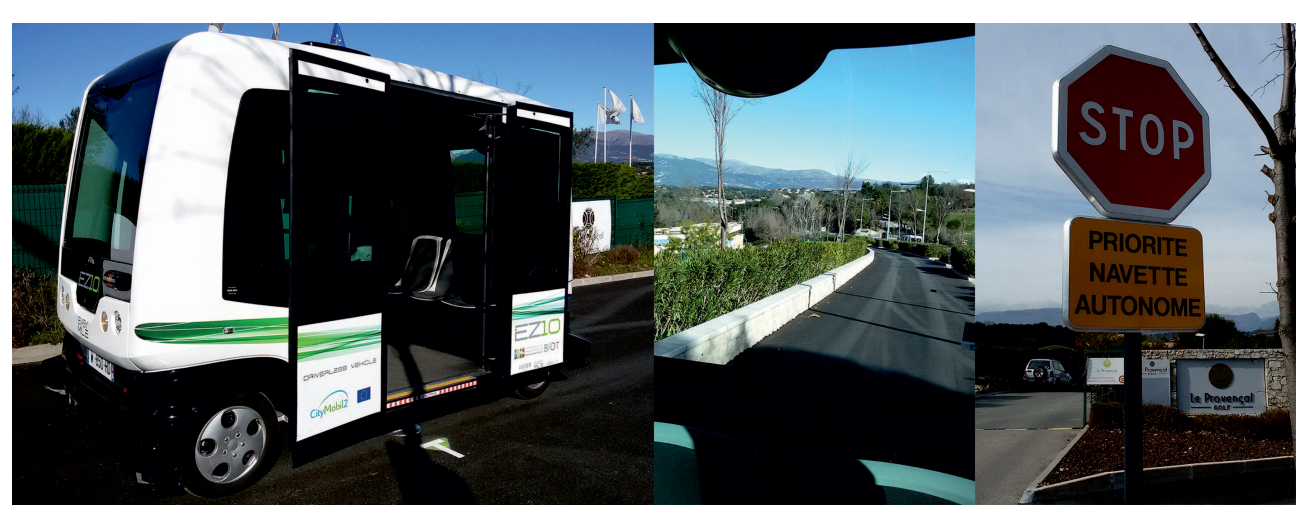

Fig. 5. EasyMile EZ10 (left), dedicated bus lane (middle), and autonomous vehicle precedence sign (right) (Sophia Antipolis, France, 2016). 
the vehicles were sending information to the control center, requesting operator's intervention.

The primary reason for the operator's presence in the autonomous vehicle was, according to the operator, the restriction imposed by applicable French legal regulations that do not allow vehicles to be admitted to streets and roads without a person authorized to drive them on board. The other reason was the occurrence of a potentially dangerous situation, due to the pilot stage of the project. In fact, this turned out to be necessary when, for example, the board computer crashed, when a situation occurred on the road that had not been foreseen in the control algorithms applied, or when an uncontrollable panic attack took place among the passengers.

During the tests, a collision of a car driven by a human and one of the unmanned vehicles occurred at the only crossing of the dedicated bus lane with a general traffic road, and as a result the presence of the operator also turned out to be necessary. Following this accident, to make the test and demonstration route more conspicuous to other road users, it was additionally marked with noticeable posters, and a STOP traffic sign with a visible note "priorité navette autonome" (French for "priority for the autonomous shuttle bus"), as shown in Fig. 5, was installed.

The tests have shown how diversified problems need to be foreseen and predicted while designing autonomous systems operating in a dynamically changing environment. Moreover, more integrated and complex information is to be extracted by means of different sensors, enabling a fast and reliable interpretation of the road traffic situation.

\subsection{Deterministic Deployment}

Deterministic deployment of nodes in WSNs is the second category identified in the process of creating physical topologies. For example, some of industrial WSNs are capable of using fixed or controllable node deployment (distribution) schemes. This type of a network would be tasked, for example, with monitoring the vibration signatures to predict maintenance needs [65]. Manually deployed sensor networks with cameras and microphones [66] are also be- ing considered for implementation. In addition, in various research projects related to natural environment monitoring, sensors are deployed manually. This makes it possible to adjust network topology to the nature of phenomena observed and to the assumptions based on which the experiments are to be carried out. As often as not the location of nodes remains deterministic (in most cases it changes slowly or remains fixed) during their service life (e.g. this is the case with investigations concerning volcanic phenomena [45]). The deployment of nodes or the range of their relocation makes it possible to prolong the operating time of a network. This can be also achieved, for example, by securing such distances between sensors that would enable the routing mechanisms applied to remain operable in the most effective way, without the need to manipulate the power of transceivers [1], [39].

In consumer applications, home automation systems (otherwise known as smart home systems) become increasingly popular. Usually, they have the features of a small-scale sensor network and take advantage of wireless communication protocols, such as Bluetooth Low Energy, ZigBee, Z-Wave and 6LoWPAN [67]. Their physical topology is usually determined at the installation stage due to the operational range being limited to just one property, whereas network communication takes place predominantly directly between the node and the base station (control center), and only occasionally with the use of intermediary network nodes [68]. In multi-family houses, radio-enabled electricity meters [69] or water meters [70] may also be found, often placed inconspicuously but effective in performing their measuring functions.

\subsubsection{Sensor Network in Cisco openBerlin Innovation Center}

The sensor network launched in Berlin, Germany, at the Cisco openBerlin Innovation Center, is an example of the deterministic deployment scheme. The network is used both as a backbone of a smart home system and as a testbed on which research projects of companies affiliated with openBerlin are evaluated [71] (Fig. 6).

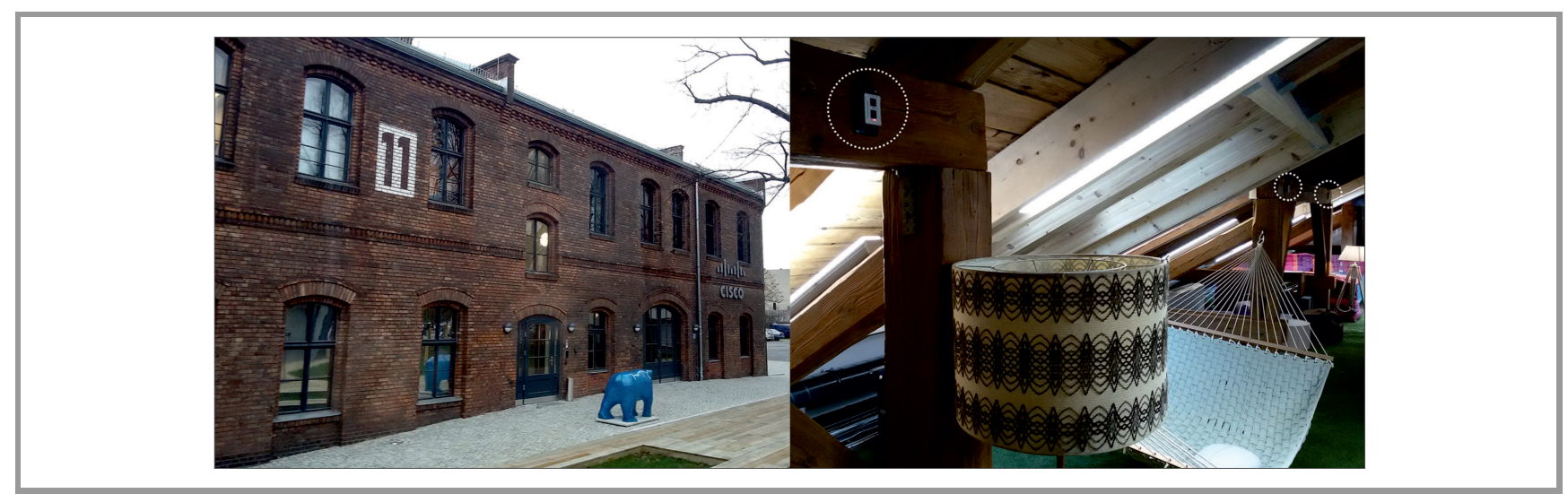

Fig. 6. Cisco openBerlin Innovation Center (left) and Bosch XDK110 based sensor network (right) (Berlin, Germany, 2016). 
The author had a chance to examine the components of the test and demonstration set, as well as to verify the topology of the network deployed within the building that consisted of several hundred nodes. The node hardware is based on the Bosch XDK platform. The devices are located at different places, including cable support ceiling systems in the office section or near ceiling joists in the recreational section, as shown in Fig. 6, and are marked with dotted circles. The Bosch XDK110 node is a hardware component equipped with a 32-bit ARM Cortex-M3 microcontroller, 1 MB Flash memory, 128 KB RAM, a Micro SD card reader, Bluetooth 4.0 Low Energy and IEEE $802.11 \mathrm{~b} / \mathrm{g} / \mathrm{n}$ modules, $560 \mathrm{mAh}$ rechargeable battery and contains 8 sensors: an accelerometer, a gyroscope, a magnetometer, as well as humidity, pressure, temperature, acoustic and light sensors. The network is used as a source of data for the system developed by an IoT company known as Relayr, and allows temperature and lighting inside the building to be controlled.

\subsubsection{Fibaro Home Automation System}

The Fibaro home automation system comprises a host controller that wirelessly manages the attached sensors and actuators (Fig. 7). The sensors include smoke, flood and motion detectors, door or window opening sensors, as well as a universal device that allows any sensor with a binary output to be added to the system. In addition, such components as a switch-key in the electric wall socket enclosure with an energy consumption measurement functionality, roller and gate shutters, lighting controller or relay switches may also be used. The system is capable of co-operating with a home weather station, wireless speakers or cameras.

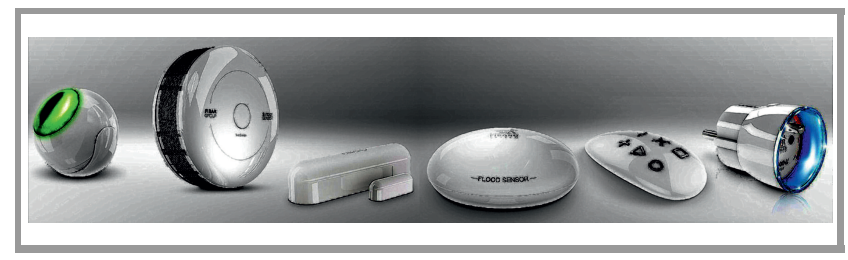

Fig. 7. Components of FIBARO home automation system.

It should be stressed that components of the Fibaro systems communicate via the Z-Wave protocol, and not via IEEE 802.15.4 ZigBee [72] that is in common use in research environments. The Z-Wave protocol was initially developed by the ZenSys, and was then largely used by the standardization organization ITU-T for the development of the G.9959 recommendation [73]. Radio communication relying on the Z-Wave protocol uses, in Europe, primarily the $868.42 \mathrm{MHz}$ frequency band, but systems for the $2.4 \mathrm{GHz}$ band are available as well. Data is routed between network nodes by assigning identifiers, whereas the throughput is not more than $40 \mathrm{kbps}$ [74]. For a commercially available device to be capable of making use of the protocol, it has to be equipped by the manufacturer with a Z-wave communication module, sold separately, and then certified for interoperability to comply with the license agreement.

\section{Conclusions}

Sensor networks continue to raise the interest of researchers who pursue further improvements to complex problems and propose potential innovative applications. Network technologies and hardware platforms are being developed in parallel, while more and more types of devices are equipped with sensor components. By striving to optimize the operation of WSNs, it is possible to make use of or draw inspiration from new ingenious concepts and products. However, despite the availability of a number of new solutions, in today's real-life sensor network deployments that enjoy an established reputation among their users and are commercially successful in urban environments, one may primarily list only those that treat the idea of WSNs in a rather simplified manner, or those that have been even developed in isolation from elaborate and complex scientific research. Hence, sensor-based systems and products are often more loosely termed as IoT, smart home, etc. This is particularly visible in the area of topology control that can be analyzed and executed as a complex and multifaceted research problem, while it is still hard to find good examples of its implementations in which it would constitute one of the main issues. It seems that, in practice, the prevailing model of implementation is empirical, i.e. draws from experiments, past experience, best practices and intuition. In some implementations, network topology is even unknowingly or deliberately pushed aside from the areas of interest or remains beyond any significant interest of network designers involved in a project - as a component of industry standards or licensed protocols implemented in building blocks relied upon.

The primary or exclusive aspect of topology control is then reduced in its essence merely to the deployment of nodes. In applications related to transport, randomness and variability of node deployment, and hence the physical topology of the network, still remain the dominant element. In applications encompassing building automation systems, in turn, it is the deterministic and static distribution of nodes established during the installation of each of the components that remains dominant.

Although sensor products and services available today still remain at different, frequently early stages of development, they allow given assumptions to be verified and lines of action to be corrected, so that they would meet the expected needs in the best possible way. This might be one of the factors facilitating the introduction of sensor or sensor-like networks into common use. This situation brilliantly illustrates the often overlooked significance of an appropriate and fruitful combination of research activities and market operability. In the area of sensor networks, a number of research investigations outpace, by decades, the current market needs or the implementation capabilities available. As a result, despite their research excellence, they might never 
be adopted in practice in their full and extensive forms. Innovative business enterprises may not be able to undertake a risk or accept costs of an implementation of too complex and seemingly expendable ideas. No wonder then that they tend to choose ready-made and easily available components and proven solutions that make it possible for them to focus on functionality issues rather than on details of all-technical aspects. This, however, is often done at the cost of getting attached to license-fee paying technologies that are not fully open and, more importantly, are developed by some other companies. As a result, business enterprises chose to provide Minimum Viable Product (MVP) as quickly as possible, i.e. such a product that would in the most favorable way satisfy the expectations of the first group of users and would allow the product to be further developed [75]. It is important then that scientists in their research efforts are able to follow the market developments and try to understand current and future needs of prospective users and, wherever possible, check and streamline their new ideas in close cooperation with operators of already existing networks and systems [76]. Moreover, socially-oriented and valuable results may be achieved when research investigations provide opportunities to transform them into real systems. Then, by gaining practical relevance, they will have a chance to enter the mainstream and be appreciated by standardization organizations, thus, at least to a certain degree, be in a position to shape the way sensor networks are implemented in the future.

\section{References}

[1] B. Musznicki and P. Zwierzykowski, "Performance evaluation of flooding algorithms for wireless sensor networks based on EffiSen: The custom-made simulator", in Simulation Technologies in Networking and Communications: Selecting the Best Tool for the Test, A.-S. K. Pathan, M. M. Monowar, and S. Khan, Eds. Boca Raton, FL, USA: CRC Press, 2015, pp. 433-458 (doi: 10.1201/b17650-21).

[2] H. Karl and A. Willig, Protocols and Architectures for Wireless Sensor Networks. Chichester: Wiley, 2005 (ISBN: 978-0-470-09510-2).

[3] I. Akyildiz and M. C. Vuran, Wireless Sensor Networks. Chichester: Wiley, 2010 (ISBN: 9780470036013).

[4] R. Faludi, Building Wireless Sensor Networks: with ZigBee, XBee, Arduino, and Processing. Sebastopol, CA, USA: O'Reilly Media, 2010 (ISBN: 978-0596807733).

[5] S. Ferdoush and X. Li, "Wireless sensor network system design using Raspberry Pi and Arduino for environmental monitoring applications", Procedia Comp. Sci., vol. 34, pp. 103-110, 2014 (doi: 10.1016/j.procs.2014.07.059).

[6] F. Leccese, M. Cagnetti, and D. Trinca, "A smart city application: a fully controlled street lighting isle based on Raspberry Pi card, a ZigBee sensor network and WiMAX", Sensors, vol. 14, no. 12, pp. 24408-24424, 2014 (doi: 10.3390/s141224408).

[7] C. P. Kruger, A. M. Abu-Mahfouz, and G. P. Hancke, "Rapid prototyping of a wireless sensor network gateway for the internet of things using off-the-shelf components", in Proc. IEEE Int. Conf. on Industr. Technol. ICIT 2015, Seville, Spain, 2015, pp. 1926-1931 (doi: 10.1109/ICIT.2015.7215378).

[8] A. D. Deshmukh and U. B. Shinde, "A low cost environment monitoring system using Raspberry Pi and Arduino with Zigbee", in Proc. Int. Conf. on Invent. Comput. Technol. ICICT 2016, Coimbatore, India, 2016, vol. 3, pp. 1-6 (doi: 10.1109/INVENTIVE.2016.7830096).
[9] A. Gupta and R. K. Jha, "A survey of 5G network: architecture and emerging technologies", IEEE Access, vol. 3, pp. 1206-1232, 2015 (doi: 10.1109/ACCESS.2015.2461602).

[10] K. Kowalik et al., "Lessons learned from WiMAX deployment at INEA", J. of Telecommun. and Inform. Technol., no. 3, pp. 34-41, 2014.

[11] B. Musznicki, K. Kowalik, P. Kołodziejski, and E. Grzybek, "Mobile and residential INEA Wi-Fi hotspot network", in 13th Int. Symp. on Wirel. Commun. Syst. ISWCS 2016, Poznań, Poland, 2016 (arXiv:1608.06606).

[12] A. Kliks, B. Musznicki, K. Kowalik, and P. Kryszkiewicz, "Perspectives for Resource Sharing in 5G Networks", Telecommun. Syst., vol. 68, no. 4, pp. 05-619, 2018 (doi: 10.1007/s11235-017-0411-3).

[13] L. Atzori, A. Iera, and G. Morabito, "From "smart objects" to "social objects": the next evolutionary step of the Internet of Things", IEEE Commun. Mag., vol. 52, no. 1, pp. 97-105, 2014 (doi: 10.1109/MCOM.2014.6710070).

[14] C. Perera, A. Zaslavsky, P. Christen, and D. Georgakopoulos, "Context aware computing for The Internet of Things: A Survey", IEEE Commun. Surv. \& Tutor., vol. 16, no. 1, pp. 414-454, 2014 (doi: 10.1109/SURV.2013.042313.00197).

[15] M. Díaz, C. Martín, and B. Rubio, "State-of-the-art, challenges, and open issues in the integration of Internet of Things and cloud computing", J. of Netw. and Comp. Appl., vol. 67, pp. 99-117, 2016 (doi: 10.1016/j.jnca.2016.01.010).

[16] J. Gubbi, R. Buyya, S. Marusic, and M. Palaniswami, "Internet of Things (IoT): A vision, architectural elements, and future directions", Future Gener. Comp. Syst., vol. 29, no. 7, pp. 1645-1660, 2013 (doi: 10.1016/j.future.2013.01.010).

[17] C. P. Kruger and G. P. Hancke, "Implementing the Internet of Things Vision in Industrial Wireless Sensor Networks", in Proc. 12th IEEE Int. Conf. on Industr. Informat. INDIN 2014, Porto Alegre, Brazil, 2014, pp. 627-632 (doi: 10.1109/INDIN.2014.6945586).

[18] A. Al-Fuqaha, M. Guizani, M. Mohammadi, M. Aledhari, and M. Ayyash, "Internet of Things: A survey on enabling technologies, protocols, and applications", IEEE Commun. Surv. \& Tutor., vol. 17, no. 4, pp. 2347-2376, 2015 (doi: 10.1109/COMST.2015.2444095).

[19] B. Musznicki and P. Zwierzykowski, "Survey of simulators for wireless sensor networks", Int. J. of Grid and Distrib.Comput., vol. 5, no. 3, pp. 23-50, 2012.

[20] M. Głąbowski, B. Musznicki, P. Nowak, and P. Zwierzykowski, "An in-depth discussion of challenges related to solving shortest path problems using ShortestPathACO based algorithms", in Information Systems Architecture and Technology; Knowledge Based Approach to the Design, Control and Decision Support, J. Świątek, L. Borzemski, A. Grzech, and Z. Wilimowska, Eds. Wrocław, Poland: Oficyna Wydawnicza Politechniki Wrocławskiej, 2013, pp. 77-88 (ISBN: 978-83-7493-802-0).

[21] M. Stein, T. Petry, I. Schweizer, M. Brachmann, and M. Mühlhäuser, "Topology control in wireless sensor networks: What blocks the breakthrough?", in Proc. 41st Conf. on Local Comp. Netw. LCN 2016, Dubai, United Arab Emirates, 2016, pp. 389-397 (doi: 10.1109/LCN.2016.67).

[22] D. Chaładyniak and J. Grzybowski, "Wybrane metody diagnozowania nieprawidłowości działania sieci teleinformatycznych", Zeszyty Naukowe Warszawskiej Wyższej Szkoty Informatyki, vol. 6, no. 8, pp. 61-76, 2012 [in Polish].

[23] M. Głąbowski, B. Musznicki, P. Nowak, and P. Zwierzykowski, "Shortest path problem solving based on ant colony optimization metaheuristic", Int. J. of Image Process. \& Commun., Special Issue: Algorithms and Protocols in Packet Networks, vol. 17, no. 1-2, pp. 7-17, 2012 (doi: 10.2478/v10248-012-0011-5).

[24] Ł. Skibniewski and J. Furtak, "Zdalne laboratorium sieciowe", Biuletyn Instytutu Automatyki i Robotyki, vol. 32, 18, pp. 3-22, 2012 [in Polish].

[25] M. Stasiak and M. Michalski, "Algorytmy wspomagające projektowanie pierścieniowych sieci optycznych", in Poznańskie Warsztaty Telekomunikacyjne PWT 2003, Poznań, Poland, 2003, pp. 121-126 [in Polish]. 
[26] P. Santi, Topology Control in Wireless Ad Hoc and Sensor Networks. Chichester: Wiley, 2005.

[27] C. Schurgers, V. Tsiatsis, S. Ganeriwal, and M. Srivastava, "Topology management for sensor networks: exploiting latency and density", in Proc. 3rd ACM Int. Symp. on Mob. Ad Hoc Network. \& Comput. MobiHoc 2002, Lausanne, Switzerland, 2002, pp. 135-145 (doi: 10.1145/513800.513817)

[28] M. Younis, I. F. Senturk, K. Akkaya, S. Lee, and F. Senel, “Topology management techniques for tolerating node failures in wireless sensor networks: A survey", Comp. Netw., vol. 58, no. 1, pp. 254-283, 2014 (doi: 10.1016/j.comnet.2013.08.021).

[29] G. Sosnowski, "Przegląd algorytmów dynamicznego zarządzania topologią w bezprzewodowych, ruchomych sieciach ad hoc", in Poznańskie Warsztaty Telekomunikacyjne PWT 2005, Poznań, Poland, 2005 [in Polish].

[30] J. Zhao and G. Cao, "Robust topology control in multi-hop cognitive radio networks", IEEE Trans. on Mob. Comput., vol. 13, no. 11, pp. 2634-2647, 2014 (doi: 10.1109/TMC.2014.2312715).

[31] K. Moon, D.-S. Yoo, W. Lee, and S.-J. Oh, "Receiver cooperation in topology control for wireless ad-hoc networks", IEEE Trans. on Wirel. Commun., vol. 14, no. 4, pp. 1858-1870, 2015 (doi: 10.1109/TWC.2014.2374617).

[32] M. A. Labrador and P. M. Wightman, Topology Control in Wireless Sensor Networks: With a Companion Simulation Tool for Teaching and Research. Springer, 2009 (ISBN: 978-1-4020-9584-9).

[33] A. A. Aziz, Y. A. Sekercioglu, P. Fitzpatrick, and M. Ivanovich, "A survey on distributed topology control techniques for extending the lifetime of battery powered wireless sensor networks", IEEE Commun. Surv. \& Tutor., vol. 15, no. 1, pp. 121-144, 2013 (doi: 10.1109/SURV.2012.031612.00124).

[34] M. Li, Z. Li, and A. Vasilakos, "A survey on topology control in wireless sensor networks: taxonomy, comparative study, and open issues", Proc. of the IEEE, vol. 101, no. 12, pp. 2538-2557, 2013 (doi: 10.1109/JPROC.2013.2257631).

[35] E. Niewiadomska-Szynkiewicz, P. Kwaśniewski, and I. Windyga, "Comparative study of wireless sensor networks energy-efficient topologies and power save protocols", J. of Telecommun. and Inform. Technol., no. 3, pp. 68-76, 2009.

[36] Y. Huang, J.-F. Martínez, V. H. Díaz, and J. Sendra, “A novel topology control approach to maintain the node degree in dynamic wireless sensor networks", Sensors, vol. 14, no. 3, pp. 4672-4688, 2014 (doi: 10.3390/s140304672).

[37] I. Yoon, D. K. Noh, and H. Shin, "Energy-aware hierarchical topology control for wireless sensor networks with energy-harvesting nodes", Int. J. of Distrib. Sensor Netw., vol. 11, no. 6, 2015 (doi: 10.1155/2015/617383).

[38] B. Chen and L. Wang, "An interference prediction-based topology control algorithm for 3-D wireless sensor networks", J. of Computat. Inform. Syst., vol. 7, no. 4, pp. 1198-1205, 2011 (doi: 10.1109/ICISE.2010.5689350).

[39] S. S. Dhillon and K. Chakrabarty, "Sensor placement for effective coverage and surveillance in distributed sensor networks, in Proc. IEEE Wirel. Commun. and Network. WCNC 2003, New Orleans, LA, USA, 2003, vol. 3 (doi: 10.1109/WCNC.2003.1200627).

[40] J. Ai and A. A. Abouzeid, "Coverage by directional sensors in randomly deployed wireless sensor networks", J. of Combinator. Optimiz., vol. 11 , no. 1 , pp. 21-41, 2006 (doi: 10.1007/s10878-006-5975-x).

[41] B. Musznicki, M. Tomczak, and P. Zwierzykowski, "Dijkstra-based localized multicast routing in wireless sensor networks", in Proc. 8th Int. Symp. on Commun. Syst., Netw. and Digit. Sig. Process. CSNDSP 2012, Poznań, Poland, 2012 (doi: 10.1109/CSNDSP.2012.6292692).

[42] M. E. M. Campista and M. G. Rubinstein, Advanced Routing Protocols for Wireless Networks. Chichester: Wiley, 2014 (ISBN: 978-1-848-21627-3).

[43] Q. Mamun, "A qualitative comparison of different logical topologies for wireless sensor networks", Sensors, vol. 12, no. 11, pp. 14887-14913, 2012 (doi: 10.3390/s121114887).
[44] X. Liu, "A survey on clustering routing protocols in wireless sensor networks", Sensors, vol. 12, no. 8, pp. 11113-11153, 2012 (doi: 10.3390/s120811113).

[45] G. Werner-Allen et al., "Deploying a wireless sensor network on an active volcano", IEEE Internet Comput., vol. 10, no. 2, pp. 18-25, 2006 (doi: 10.1109/MIC.2006.26).

[46] H. Zhang and J. C. Hou, "Is deterministic deployment worse than random deployment for wireless sensor networks?", in Proc. 25th IEEE Int. Conf. on Comp. Commun. INFOCOM 2006, Barcelona, Spain, 2006, pp. 1-13 (doi: 10.1109/INFOCOM.2006.290).

[47] S. N. Simić and S. Sastry, "Distributed environmental monitoring using random sensor networks" in Information Processing in Sensor Networks: Second International Workshop, IPSN 2003, Palo Alto, CA, USA, April 2003, Proceedings, F. Zhao and L. Guibas, Eds. LNCS, vol. 2634, pp. 582-592. Berlin Heidelberg: Springer, 2003.

[48] K. Römer and F. Mattern, "The design space of wireless sensor networks", IEEE Wireless Commun., vol. 11, no. 6, pp. 54-61, 2004 (doi: 10.1109/MWC.2004.1368897).

[49] A. Howard, M. J. Matarić, and G. S. Sukhatme, "An incremental self-deployment algorithm for mobile sensor networks", Autonomous Robots, vol. 13, no. 2, pp. 113-126, 2002 (doi: 10.1023/A:1019625207705).

[50] H. Gao et al., "High speed data routing in vehicular sensor networks", J. of Commun., vol. 5, no. 3, pp. 181-188, 2010 (doi: 10.4304/jcm.5.3.181-188).

[51] M. Wooldridge and N. R. Jennings, "Intelligent agents: theory and practice", The Knowl. Engin. Rev., vol. 10, no. 2, pp. 115-152, 1995 (doi: 10.1017/S0269888900008122).

[52] U. Lee and M. Gerla, "A survey of urban vehicular sensing platforms", Computer Netw., vol. 54, no. 4, pp. 527-544, 2010 (doi: 10.1016/j.comnet.2009.07.011).

[53] "IEEE Standard for Information technology - Telecommunications and information exchange between systems - Local and metropolitan area networks - Specific requirements, Part 11: Wireless LAN Medium Access Control (MAC) and Physical Layer (PHY) Specifications", IEEE Std 802.11 ${ }^{\mathrm{TM}}$-2012, 29 March 2012 [Online]. Available: https://www.iith.ac.in/ tbr/teaching/docs/802.11-2007.pdf

[54] K. Kowalik, A. Kliks, B. Musznicki, M. Kołodziejski, and P. Kryszkiewicz, "Observation of WiMAX Radio Parameters to Enhance Spectrum Utilisation in Mixed Environment", J. of Telecommun. and Inform. Technol., no. 1, pp. 42-50, 2018 (doi: 10.26636/jtit.2018.123917).

[55] H. Conceição, M. Ferreira, and J. Barros, "On the urban connectivity of vehicular sensor networks", in Distributed Computing in Sensor Systems, 4th IEEE International Conference, DCOSS 2008, Santorini Island, Greece, June 11-14, 2008, Proceedings, S. E. Nikoletseas, B. S. Chlebus, D. B. Johnson, and B. Krishnamachari, Eds. LNCS, vol. 5067, pp. 112-125. Berlin Heidelberg: Springer, 2008 (doi: 10.1007/978-3-540-69170-9_8).

[56] M. Boban, T. T. Vinhoza, M. Ferreira, J. Barros, and O. K. Tonguz, "Impact of vehicles as obstacles in vehicular ad hoc networks", IEEE J. on Selec. Areas in Commun., vol. 29, no. 1, pp. 15-28, 2011 (doi: 10.1109/JSAC.2011.110103).

[57] A. Cardote, S. Sargento, and P. Steenkiste, "On the connection availability between relay nodes in a VANET", in Proc. IEEE Globecom Worksh. GC Wkshps 2010, Miami, FL, USA, 2010, pp. 181-185 (doi: 10.1109/GLOCOMW.2010.5700255).

[58] C. Ameixieira et al., "HarborNet: A real-world testbed for vehicular networks", IEEE Commun. Mag., vol. 52, no. 9, pp. 108-114, 2014 (doi: 10.1109/MCOM.2014.6894460).

[59] "Creating The World's Largest Network of Connected Vehicles for Smart Cities" [Online]. Available: https://www.worldwifiday.com/ wp-content/uploads/2016/05/3.-PortoCaseStudy_Letter_2016-0415.pdf

[60] "IEEE Standard for Information technology - Telecommunications and information exchange between systems - Local and metropolitan area networks - Specific requirements", IEEE Std 802.11 $\mathrm{p}^{\mathrm{TM}}-2010$, 15 July 2010. 
[61] R. C. Shah, S. Roy, S. Jain, and W. Brunette, "Data MULEs: Modeling and Analysis of a Three-tier Architecture for Sparse Sensor Networks", Ad Hoc Netw., vol. 1, no. 2, pp. 215-233, 2003 (doi: 10.1016/S1570-8705(03)00003-9).

[62] P. Santos et al., "Demo abstract: Experiments on using vehicles as data mules for data collection from urban sensors", in Proc. 12th Eur. Conf. on Wirel. Sensor Netw. EWSN 2015, Porto, Portugal, 2015, pp. 17-18.

[63] "CityMobil2 Experience and Recommendations" [Online]. Available: https://www.polisnetwork.eu/ CityMobil2\%20booklet\%20web\%20final_17\%2011\%202016.pdf

[64] A. Alessandrini, A. Cattivera, C. Holguin, and D. Stam, "CityMobil2: Challenges and opportunities of fully automated mobility" in Road Vehicle Automation, G. Meyer and S. Beiker, Eds. Springer, 2014, pp. 169-184 (doi: 10.1007/978-3-319-05990-7).

[65] L. Krishnamurthy et al., "Design and deployment of industrial sensor networks: experiences from a semiconductor plant and the North Sea", in Proc. 3rd Int. Conf. on Embed. Network. Sensor Syst. SenSys 2005, San Diego, CA, USA, 2005, pp. 64-75 (doi: 10.1145/1098918.1098926).

[66] J. Yick, B. Mukherjee, and D. Ghosal, "Wireless sensor network survey", Computer Netw., vol. 52, no. 12, pp. 2292-2330, 2008 (doi: 10.1016/j.comnet.2008.04.002).

[67] C. Gomez and J. Paradells, "Wireless home automation networks: A survey of architectures and technologies", IEEE Commun. Mag., vol. 48 , no. 6 , pp. $92-101,2010$ (doi: 10.1109/MCOM.2010.5473869).

[68] T. Zachariah, N. Klugman, B. Campbell, J. Adkins, N. Jackson, and P. Dutta, "The Internet of Things has a gateway problem", in Proc. 16th Int. Worksh. on Mobile Comput. Syst. and Appl. HotMobile 2015, Santa Fe, New Mexico, USA, 2015, pp. 27-32 (doi: 10.1145/2699343.2699344).

[69] V. C. Gungor, D. Sahin, T. Kocak, S. Ergut, C. Buccella, C. Cecati, and G. P. Hancke, "Smart grid technologies: Communication technologies and standards", IEEE Trans. on Industr. Informat., vol. 7, no 4, pp. 529-539, 2011 (doi: 10.1109/TII.2011.2166794).

[70] L. Quan-Xi and L. Gang, "Design of remote automatic meter reading system based on ZigBee and GPRS", in Proc. 3rd Int. Symp. on Comp. Sci. and Computat. Technol. ISCSCT 2010, Jiaozuo, China, 2010, vol. 2, pp. 186-189.

[71] Cisco openBerlin Innovation Center [Online]. Available: https://www.cisco.com/c/m/de_de/innovationcenter/berlin.html

[72] "IEEE Standard for Local and metropolitan area networks - Part 15.4: Low-Rate Wireless Personal Area Networks (LR-WPANs)", IEEE Std 802.15.4-2011, 5 September 2011 [Online]. Available: https://standards.ieee.org/standard/802_15_4-2011.html
[73] "Series G: Transmission Systems and Media, Digital Systems and Networks - Access networks - In premises networks - Short range narrow-band digital radiocommunication transceivers - PHY, MAC, SAR and LLC layer specifications", Recommendation ITU-T G.9959, 2015 [Online]. Available: https://www.itu.int/rec/ T-REC-G.9959/en

[74] C. Gomez and J. Paradells, "Wireless home automation networks: A survey of architectures and technologies", IEEE Commun. Mag., vol. 48, no. 6, pp. 92-101, 2010 (doi: 10.1109/COM.2010.5473869).

[75] D. R. Moogk, "Minimum viable product and the importance of experimentation in technology startups", Technol. Innov. Manag. Rev., vol. 2, no. 3, pp. 23-26, 2012 (doi: 10.22215/timreview/535).

[76] P. Walkowiak, R. Szalski, B. Musznicki, D. Dudek, K. Kowalik, and P. Zwierzykowski, "Evaluation of CARMNET System in INEA HOTSPOT Network", in Proc. IEICE Inform. and Commun. Technol. Forum ICTF 2014, Poznań, Poland, 2014 (doi: 10.13140/2.1.2751.4567).

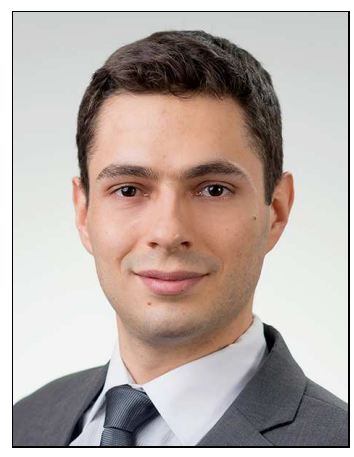

Bartosz Musznicki has been expanding his experience in the area of management and network architecture at INEA a telecommunications operator active in the Greater Poland region. He is currently working for Capgemini in one of Europe's major commercial network projects. He is pursuing Ph.D. at the Poznan University of Technology, Poland. His main research interests include topology control and routing in wireless sensor networks. $\mathrm{He}$ is an author of four book chapters, eight journal articles and six conference papers.

(iD) https://orcid.org/0000-0002-7529-8898

E-mail: rsrch@musznicki.com

Chair of Communication and Computer Networks

Faculty of Electronics and Telecommunications

Poznan University of Technology

Polanka 3

61-131 Poznań, Poland 